\title{
BMJ Open Eosinopenia as a biomarker for antibiotic use in COPD exacerbations: protocol for a retrospective hospital- based cohort study
}

\author{
Mei Yang, ${ }^{1}$ Xuemei Liu, ${ }^{1,2}$ Qiongqiong Hu, ${ }^{3}$ Junjie Li,,${ }^{1}$ Sijia Fu, ${ }^{1}$ Daohong Chen, \\ Yanqing Wu, ${ }^{1}$ Ai Luo, ${ }^{1}$ Xiawei Zhang, ${ }^{4}$ Ruizhi Feng, ${ }^{1}$ Guo Xu, ${ }^{1}$ Can Liu, ${ }^{1}$ \\ Hongli Jiang, ${ }^{1}$ Wei Liu (D) ${ }^{1}$
}

To cite: Yang M, Liu X, Hu Q, et al. Eosinopenia as a biomarker for antibiotic use in COPD exacerbations: protocol for a retrospective hospitalbased cohort study. BMJ Open 2022;12:e051939. doi:10.1136/ bmjopen-2021-051939

- Prepublication history for this paper is available online To view these files, please visit the journal online (http://dx.doi org/10.1136/bmjopen-2021 051939).

Received 02 April 2021 Accepted 10 January 2022

Check for updates

(c) Author(s) (or their employer(s)) 2022. Re-use permitted under CC BY-NC. No commercial re-use. See rights and permissions. Published by BMJ.

For numbered affiliations see end of article.

Correspondence to

Dr Wei Liu;

liuwei0929@wchscu.cn

\section{ABSTRACT}

Introduction The acute exacerbation of chronic obstructive pulmonary disease (AECOPD) has a seriously negative impact on patients' healths condition and disease progression. Bacterial infection is closely related to $A E C O P D$, and antibiotics are frequently used in clinical practice. The lack of specific biomarkers for rational antibiotics use always leads to antibiotics abuse in chronic obstructive pulmonary disease (COPD) flareups. Eosinopenia has been considered to be related to increased bacterial load of potentially pathogenic organisms at the onset of COPD exacerbations. Therefore, this study aims to investigate whether eosinopenia could be used as a reference for the use of antibiotics in AECOPD.

Methods and analysis In this study, a hospital-based retrospective cohort design will be adopted to analyse the clinical data of inpatients who are primarily diagnosed with AECOPD in West China Hospital of Sichuan University from 1 January 2010 to 31 December 2020. Relevant data will be extracted from the Clinical Big Data Platform for Scientific Research in West China Hospital, including demographic characteristics, blood eosinophil count, procalcitonin, $\mathrm{C}$ reactive protein, microbial cultivation, antibiotics use, length of hospital stay, non-invasive ventilation use, intensive care unit transfer and mortality, etc. The collected data will be described and inferred by corresponding statistical methods according to the data type and their distributions. Multiple binary logistic regression models will be used to analyse the relationship between blood eosinophil count and bacterial infection. The antibiotics use, and patient morbidity and mortality will be compared between patients with or without eosinopenia.

Ethics and dissemination This study has been approved by the Biomedical Ethics Review Board of West China Hospital of Sichuan University (Approval No. 2020-1056). And the research results will be published in a peerreviewed journal.

Trial registration number ChiCTR2000039379.

\section{INTRODUCTION}

Chronic obstructive pulmonary disease (COPD) is a common respiratory disease that
Strengths and limitations of this study

- This is a pioneering study to comprehensively reveal the role of blood eosinophils in the treatment and prognosis of chronic obstructive pulmonary disease exacerbations by exploring its relationship with bacterial infection, antibiotics use, and patients' morbidity and mortality.

- This study adopts a retrospective cohort study design involving a relatively large representative sample, which gives more reliable results with greater precision and power.

- The data of this study comes from real clinical evidence, and the process of data generation and collection is consistent with the actual clinical medical practice.

- The data may be incomplete and inaccurate due to the development of time, a short period of observation, the insensitivity and unspecificity of disease coding, and the inaccuracy of clinical recordings.

is responsible for considerable and growing morbidity, mortality and healthcare expenses worldwide. $^{12}$ Acute exacerbation of COPD (AECOPD) that characterised by acute worsening of cough and mucus production and marked gas trapping is closely related to worse health conditions, and increased hospitalisations, readmissions and fatality rate. ${ }^{3}$ Minimising the negative impact of the current exacerbation and preventing the development of subsequent events are principal goals of AECOPD treatment. ${ }^{4}$

COPD exacerbations are heterogeneous events that are now thought to be caused by complex interactions among the host, airway bacteria, respiratory viruses and environmental pollution, leading to an increase in the inflammatory burden. ${ }^{5}$ Some studies demonstrated that COPD exacerbations are triggered commonly by respiratory viruses and bacteria, which infect the lower airway 
and increase airway inflammation. ${ }^{5-7}$ It has been found that $40 \%-55 \%$ of COPD exacerbations are associated with bacterial infections. ${ }^{8}{ }^{9}$ COPD flare-ups caused by bacterial infections are characterised by a more severe airway and systemic inflammation responses compared with those caused by non-bacterial infections. ${ }^{10}$ Timely initiation of antibiotic therapy helps to reduce in-hospital mortality, treatment failure rate and rehospitalisations and to prolong the time to the next attack. ${ }^{11}$ In consequence, antibiotics prophylaxis or empirical antibiotics treatment is heavily used in the early stage of flare-ups, especially in those with severe exacerbations. ${ }^{12}$ However, it is critical to note that AECOPD episodes may also be triggered by non-bacterial factors such as viral infections and smoking exposures. ${ }^{13}$ Therefore, antibiotic therapy without a verification of bacterial infection leads to a grim overuse of antibiotics in the AECOPD population. ${ }^{14}$ A study in France has shown that antibiotic prescriptions attributable to viral lower respiratory tract infections account for $20 \%$ of outpatient antibiotic use in the elderly. ${ }^{15}$ The considerable drawbacks of antibiotics abuse have been highlighted concerning the possible adverse effects, increased antibiotics resistance and heavy financial burden. ${ }^{16}{ }^{17}$ Besides, antibiotics abuse also leads to a delay in diagnosis as signs and symptoms of bacterial infections may disappear. ${ }^{18}$ Appropriate antibiotics prescribing decisions made by healthcare professionals will not only help the management of current exacerbations, but also reduce aforesaid risks. Therefore, the exploration for biomarkers that can reliably predict bacterial infection and indicate antibiotics use in AECOPD patients is in great demand. However, it is still difficult to accurately identify bacterial infection in clinical practice under the direction of current indicators. ${ }^{919}$ The effect of common biomarkers, such as serum procalcitonin (PCT) and $\mathrm{C}$ reactive protein $(\mathrm{CRP})$, on distinguishing causative pathogens during COPD exacerbation is limited as they alone are not sufficient to confirm a bacterial infection diagnosis. ${ }^{20}$ And it is also difficult to implement CRP and PCT test in some primary care hospitals.

Normal eosinophil percentage is between $3 \%$ and $5 \%$ in the peripheral blood, equating to an absolute peripheral eosinophil count of $0.35-0.5 \times 10^{9} / \mathrm{L} .^{21}{ }^{22}$ Previous studies have shown that blood eosinophil count (BEC) decreases in patients with COPD exacerbations with an increase of sputum bacterial counts. ${ }^{23-25}$ Also, a study has shown that $2 \%$ of BEC may be considered as an indicator to distinguish bacterial from non-bacterial infections in AECOPD patients. ${ }^{26}$ What is more, BEC returns to the normal level faster than serum CRP and PCT under appropriate antibiotic therapy, ${ }^{11}$ suggesting a high sensitivity of eosinopenia in indicating bacterial infection and its potential role in indicating antibiotic therapy.

\section{STUDY OBJECTIVES}

This study is primarily designed to explore that whether the level of peripheral blood eosinophils could be used as a reliable biomarker to predict bacterial infection and antibiotic treatment in hospitalised patients with AECOPD. This study will potentially guide the management strategies and contribute to the development of precision medicine in COPD. Three specific clinical and scientific issues will be addressed as follows:

1. To explore the association between blood eosinopenia and bacterial infection in hospitalised patients with AECOPD.

2. To indicate the role of blood eosinopenia as a reference for antibiotics use in hospitalised patients with AECOPD.

3. To examine the association between blood eosinopenia and prognosis in hospitalised patients with AECOPD.

\section{METHODS AND ANALYSIS}

\section{Study design, setting and data sources}

This retrospective cohort study will be conducted in West China Hospital of Sichuan University, a national medical centre in western China and a referral hospital with more than 270000 patients discharged each year. Data of this study will include health records in a 11-year period from 1 January 2010 to 31 December 2020 from the Clinical Big Data Platform for Scientific Research (CBDPSR). CBDPSR is a hospital-based network that integrates 13 health information systems, 4.8 billion medical records and 72443800 medical visits. It is focused on improving healthcare deliveries, health outcomes and medical researches. This protocol is reported according to the Strengthening the Reporting of Observational Studies in Epidemiology checklist. ${ }^{27}$

Data extraction will be completed by two experienced respiratory clinicians and two senior engineers from the Information Centre of West China Hospital. Relevant data will be extracted from medical charts and electronic medical records, and re-entered into a research database. Further quality control for data consistency and cleaning will be performed. As some data may be unrecorded or incomplete in the database, patients or their family members will be contacted by telephone to obtain the missing data. Otherwise, the data will be removed from the database. Natural language processing and text information structuring will be used to clean up imaging tests and lung function tests because of the enormous amount of data. Figure 1 shows the flow chart of the study.

\section{Study participants}

This study will include inpatients (18-80 years old) admitted to West China Hospital from 1 January 2010 to 31 December 2020 and discharged with a primary diagnosis of AECOPD. The presence of AECOPD will be identified based on the International Classification of Diseases-10 (ICD-10). Patients with a primary diagnosis of ICD-10 code of J44.0 or J44.1 will be included in the study. An initial screening began on March 1, 2021, and yielded 16219 records. We will only collect inpatient data for analysis because of the large clinical differences between 


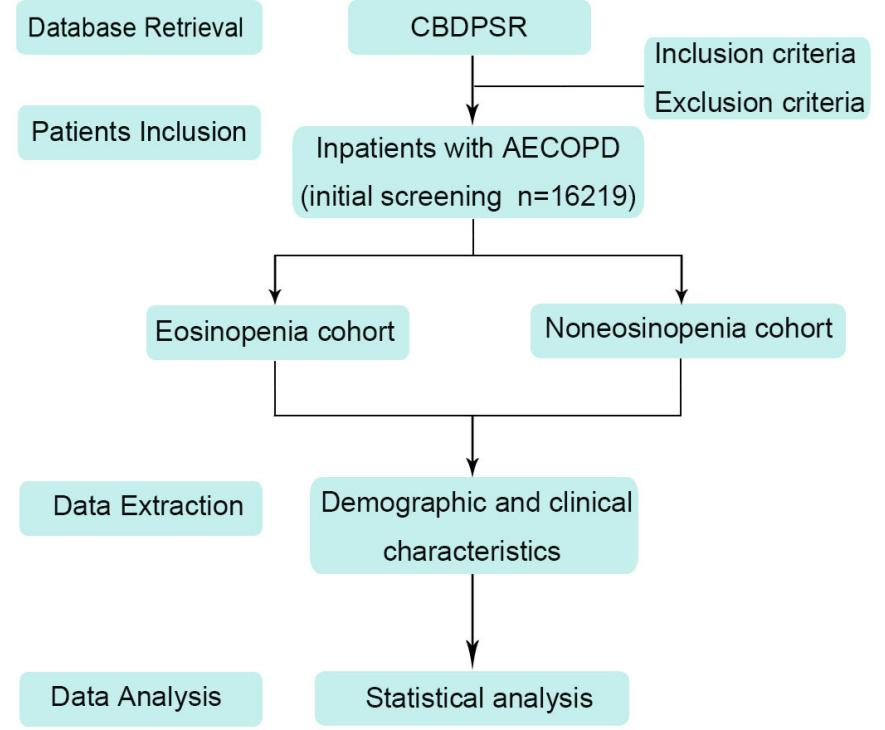

Figure 1 The flow chart of the study. AECOPD, acute exacerbation of chronic obstructive pulmonary disease; CBDPSR, Clinical Big Data Platform for Scientific Research.

outpatients and inpatients, as well as an easier access to inpatient information. Patients will be excluded if:

1. They have a different primary diagnosis other than AECOPD at discharge, such as pulmonary tuberculosis, bronchiectasis, pulmonary fibrosis, acute pulmonary embolism, acute pulmonary oedema, pneumothorax or arrhythmia.

2. They have complications that may affect the BEC, such as asthma, leucocythaemia, parasite infection, allergic disorders, eczema, eosinophilic pneumonia, solid tumours, severe immunodeficiency disease or autoimmune diseases.

3. They have severe liver, heart or renal failures.

4. They have incomplete clinical records for analysis, for example, lack of blood routine tests.

\section{Patient and public involvement}

As this research is a retrospective study, it does not involve patient recruitment and signing of informed consent. Patients and the public are not involved in the development of the research question, outcome measures, study design, study conduct, as well as study reporting.

\section{Outcomes}

Demographic and clinical characteristics, including age, sex, height, weight, course of disease, diagnosis, comorbidities (gastroesophageal reflux disease, coronary heart disease, hypertension, diabetes, osteoporosis, cerebrovascular accident, anxiety and depression), symptoms, smoking history and pack-years, severity of airway limitation based on the Global Initiative for Chronic Obstructive Lung Disease (GOLD) guideline, length of hospital stay, intensive care unit (ICU) admission, length of ICU stay, total cost, in-hospital mortality, antibiotic therapy, adjunctive therapy, invasive ventilator or non-invasive ventilator use, AECOPD associated hospital admissions during the previous year, readmissions within 30 days after discharge, pulmonary function test and imaging examination, will be collected.

Data from laboratory tests that conducted within 24 hours after admission, including serum PCT, CRP and IL-6, bacterial culture, white cell count (WCC), BEC, blood eosinophil percentage, blood neutrophil count (BNC), blood neutrophil percentage (BNP) and arterial blood gas analysis, will be collected. Culture results using samples of sputum or broncho alveolar lavage (BAL) fluid will be recorded for bacterial infection identification. In cases with positive bacterial cultures, the name of pathogens and antibiotic sensitivity results will be extracted. However, bacterial infections in blood, extrapulmonary organs or tissues are supposed to be excluded. Results of nucleic acid test, antibody test, (1-3)- $\beta$-d-glucan assay, galactomannan assay and cultivation in valid samples of sputum and BAL fluid will also be collected to identify the viral and fungal infections. The date of chest X-ray and CT scan will be collected, along with the findings. Where possible, a radiologist or a senior physician will be involved in the image reading. Serum PCT, CRP and leucocyte count are commonly used acute phase reactants in bacterial infections. CRP and leucocyte count do not have sufficient specificity in differentiating bacterial infections from noninfectious inflammation and viral infections. PCT is a precursor of the hormone calcitonin, and it has been accepted that PCT is specific to bacterial infections. ${ }^{28}$ Some studies have shown that PCT can effectively distinguish patients with bacterial AECOPD from those with non-bacterial AECOPD and guide the rational use of antibiotics. ${ }^{28-30}$ And PCT is a better marker for predicting positivity of sputum culture compared with CRP especially in severe and hospitalised patients with AECOPD who require ICU or mechanical ventilation support. ${ }^{30}$ Therefore, in this study, bacterial infection will be defined as positive pathogenic bacterial cultures in valid sputum or BAL fluid with increased serum PCT or infectious lesions in imaging results with clinical manifestations with increased sputum purulence.

Information of antibiotic therapy will include whether antibiotics are prescribed during hospitalisation, details about individual antibiotics (eg, name, route, dosage, duration of antibiotic treatment, frequency, time to the first antibiotic prescription from admission) and whether double or triple antibiotic therapy is used. Duration of antibiotic treatment will be defined as days with any antibiotics exposure for respiratory infections during hospitalisation. Antibiotic treatment for infections in other parts of the body will not be recorded. We will define antibiotic therapy as oral or intravenous antibiotics use for five or more consecutive days. Adjunctive therapy will include commonly used medications such as inhaled bronchodilator, theophylline, systemic corticosteroids, maintenance inhaled corticosteroids (ICS) and aerosol ICS during hospitalisation. These therapies will be recorded on the name, route, dosage, duration of treatment, frequency and time to the first prescription from admission. We 
define maintenance ICS use as regular ICS use for three or more consecutive months before admission.

\section{Sample size calculation}

According to the previous study, ${ }^{26}$ the incidence of bacterial infection was $20 \%$ in the noneosinopenia group and $30 \%$ in eosinopenia group, using $2 \%$ as the cut-off in blood routine examination. We used a CI of $95 \%$ and a p value of 0.05 to achieve $85 \%$ power to detect a difference between the two cohorts. An estimated 1:3 ratio between the two groups was selected given that eosinopenia cases are more common in the AECOPD population. ${ }^{26} 31$ The estimated sample size is 948 , with 711 in the eosinopenia group, and 237 in the non-eosinopenia group.

\section{Proposed statistical analysis}

Patients will be divided into two cohorts with or without eosinopenia according to the level of peripheral eosinophils in blood routine examination. We will use $2 \%$ or $0.2 \times 10^{9} / \mathrm{L}$ as the threshold. ${ }^{26}$ Baseline demographic characteristics including age, sex, course of disease, smoking history and pack-years, body mass index (BMI) and clinical information including comorbidity, the severity of airway limitation based on the GOLD guideline will be summarised using descriptive statistics. Quantitative variables including age, PCT, CRP, IL-6, BMI, course of disease, length of hospital stay, length of ICU stay, dose and duration of treatment, AECOPD associated hospital admissions during the previous year, total cost, WCC, BEC and BNC, with a unimodal symmetric distribution, especially those with a normal distribution or an approximate normal distribution, will be summarised using mean $\pm \mathrm{SD}$, and those with an asymmetric distribution or unclear distribution will be summarised using median and IQR. Categorical variables including sex, smoking history, invasive ventilator or non-invasive ventilator use, ICU admission, in-hospital mortality, antibiotics use, corticosteroids use, bronchodilator use, readmission within 30 days after discharge, positive findings in imaging examination and bacterial culture, will be summarised using frequencies and percentages. Where appropriate, 95\% CIs will be provided. The $\chi^{2}$ test or Fisher's exact test will be performed for the categorical variables. Student's t-test and Mann-Whitney $\mathrm{U}$ test will be used for continuous normally and abnormally distributed variables, respectively.

We will identify the effect of blood eosinopenia, with or without other potential covariates, on predicting a bacterial infection by conducting binary logistic regressions. As informed by prior literature, ${ }^{32-34}$ the potential covariates in predicting bacterial infection will include but not limited to : (1) age; (2) sex; (3) WCC; (4) BNP; (5) CRP; (6) PCT; (7) IL-6; (8) use of systemic corticosteroids; (9) use of maintenance ICS, (10) GOLD stage and (11) presence of chronic comorbidities. WCC, BNP, CRP, PCT and IL-6 will be converted into binary variables based on the optimal cut-off values, which are 10000 cells $/ \mathrm{mm}^{3}, 75 \%$, $5 \mathrm{mg} / \mathrm{L}, 0.046 \mathrm{ng} / \mathrm{mL}$, and $7 \mathrm{pg} / \mathrm{mL}$, respectively. The effect of individual predictors on dependent variables will be reported as adjusted ORs with 95\% CIs. The logistic regression analysis will be assessed by Hosmer-Lemeshow test. Multivariate analysis will be performed in different models that include different covariates.

Subgroup analyses will be performed to determine whether the association between blood eosinophils and antibiotic therapy is modified by maintenance ICS use and other confounders. Sensitivity analyses will be undertaken to determine the robustness of the association. First, since the cut-off of eosinopenia varies from $0.01 \%$ to $4 \%$ in previously reported studies, ${ }^{25}{ }^{35-38}$ we will analyse that whether the association changes when different cut-off values are used to define peripheral blood eosinopenia. Second, we will use positive bacterial cultures accompanied with increased serum PCT and purulent sputum as the evidence of respiratory bacterial infection to explore the impact of bacterial infection definition on the association. Third, we will assess the association exclusively in patients with a confirmed diagnosis of AECOPD that supported by a reported obstructive spirometry.

All analyses will be performed using SPSS V.22.0, and a $\mathrm{p}<0.05$ is considered statistically significant.

\section{DISCUSSION}

Acute exacerbation is a critical component in the clinical course of COPD and is associated with significant morbidity and mortality. It presents a clinical complexity for clinicians and a disproportionate burden on healthcare resources. ${ }^{39}$ Current evidence indicates that the incidence of bacterial exacerbation is approximately $50 \%$ in AECOPD episodes, which is even higher in those with advanced COPD. ${ }^{40}$ Hence, empirical antibiotic therapy is preferred, especially in hospitalised AECOPD patients, as timely antibiotic treatment is beneficial not only in the current attack by promoting a rapid resolution of acute symptoms and decreasing respiratory-related deaths, ${ }^{41}$ but also within the next few months by extending the exacerbation-free intervals. ${ }^{42}$ However, since viral infections and non-infectious factors can be also involved in the onset of AECOPD, non-controlled use of antibiotics can be associated with increased treatment failures, bacterial resistance and even chances of relapse. ${ }^{42}$ Therefore, rational antibiotic therapy is of great importance in clinical practice, and it is largely based on the appropriate and timely identification for bacterial infections.

Some guidelines recommend antibiotic treatment based on respiratory symptoms and increased purulence in particular. ${ }^{43}{ }^{44}$ However, sputum colour alone may not be sufficient to identify patients possibly benefiting from antibiotics. ${ }^{45}$ Results from previous studies have emphasised the use of CRP and PCT as predictors in antibiotics prescription in general practice. ${ }^{46} 47$ They are, however, not yet available as point-of-care tests in primary care. Some scoring systems such as systemic inflammatory response syndrome score and CRB-65 score may identify severe AECOPD at high risk of bacterial infections, but 
no reports have supported their roles as indicators for antibiotic treatment. ${ }^{48-50}$

Previous studies have shown that AECOPD patients with a low BEC have a poorer prognosis, higher risk of bacterial infection, longer hospital stay and increased mortality than those without. ${ }^{2638}$ This study will be a pioneering retrospective study to identify the role of eosinopenia as a reference for antibiotic treatment, and the characteristics of patients who are most likely to benefit from prescribing antibiotics in hospitalised patients with AECOPD. Our results will highlight the issue of antibiotics use in AECOPD, and also raise awareness among other infective diseases. A prospective multicentre study may be required to confirm the results and to provide more consolidate grounds for the role of blood eosinophils in guiding COPD treatment.

There are also some limitations in our study. First, we will collect clinical data from a ready-made database. The clinical recordings may be incomplete and inaccurate, and the disease coding may be insensitive and unspecific. Second, the time span of our cohort is over 11 years, and the techniques of nucleic acid test for pathogen detection have achieved a great development during the recent decade. Therefore, the nucleic acid results will be recorded based on different techniques. As newly-developing techniques such as next-generation sequencing are generally more sensitive than conventional ones such as traditional PCR, some results from early years could be missed and less accurate (false negative or false positive).

\section{ETHICAL APPROVAL AND DISSEMINATION}

The data in our study are available on request. Patient privacy records and information are anonymous and hidden before analysis. Results of the study will be presented in a peer-reviewed publication. We will strictly follow ethical guidelines such as the Helsinki Declaration of the World Medical Congress and the Ethical Review of Biomedical Research Involving Humans (Trial). This study has been approved by the Biomedical Ethics Review Board of West China Hospital of Sichuan University (Approval No. 2020-1056).

\section{Author affiliations}

${ }^{1}$ Division of Pulmonary Diseases, Department of Integrated Traditional and Western Medicine, Sichuan University West China Hospital, Chengdu, Sichuan, China ${ }^{2}$ Department of Pulmonary Diseases, State Key Laboratory of Biotherapy of China, Sichuan University West China Hospital, Chengdu, Sichuan, China

${ }^{3}$ West China School of Medicine, Sichuan University West China Hospital, Chengdu, Sichuan, China

${ }^{4}$ Respiratory Medicine Unit and National Institute for Health Research, Nuffield Department of Medicine Experimental Medicine, Oxford Biomedical Research Centre, University of Oxford, Oxford, UK

Acknowledgements Special thanks to the West China Hospital Information Centre of Sichuan University for technical support in data selection.

Contributors MY and XL were first coauthors and they contributed equally to this work. WL and $\mathrm{HJ}$ designed the study, drafted and revised the manuscript. WL, MY and XL contributed to the trial registration, ethics application, data preliminary screening and manuscript writing. QH, JL, DC, SF, YW, GX, CL, AL and
$\mathrm{XZ}$ participated in the protocol development and data preliminary screening. RF provided expertise for data collection. All authors have read and approved the final manuscript. WL is the study guarantor.

Funding This work is financially supported by the National Natural Science Foundation of China (grant number 81900039).

Disclaimer The funder has no role in the design of the study and writing of the manuscript and will not involve in the collection, analysis, and interpretation of data.

Competing interests None declared.

Patient and public involvement Patients and/or the public were not involved in the design, or conduct, or reporting, or dissemination plans of this research.

Patient consent for publication Not applicable.

Provenance and peer review Not commissioned; externally peer reviewed.

Open access This is an open access article distributed in accordance with the Creative Commons Attribution Non Commercial (CC BY-NC 4.0) license, which permits others to distribute, remix, adapt, build upon this work non-commercially, and license their derivative works on different terms, provided the original work is properly cited, appropriate credit is given, any changes made indicated, and the use is non-commercial. See: http://creativecommons.org/licenses/by-nc/4.0/.

ORCID iD

Wei Liu http://orcid.org/0000-0001-6929-5984

\section{REFERENCES}

1 Vestbo J, Hurd SS, Agustí AG, et al. Global strategy for the diagnosis, management, and prevention of chronic obstructive pulmonary disease: gold executive summary. Am J Respir Crit Care Med 2013;187:347-65.

2 Mathers CD, Loncar D. Projections of global mortality and burden of disease from 2002 to 2030. PLoS Med 2006;3:e442.

3 Ortega $\mathrm{H}$, Llanos J-P, Lafeuille $\mathrm{M}-\mathrm{H}$, et al. Burden of disease associated with a COPD eosinophilic phenotype. Int J Chron Obstruct Pulmon Dis 2018;13:2425-33.

4 Martinez FJ, Han MK, Flaherty K, et al. Role of infection and antimicrobial therapy in acute exacerbations of chronic obstructive pulmonary disease. Expert Rev Anti Infect Ther 2006;4:101-24.

5 Wedzicha JA, Seemungal TAR. Copd exacerbations: defining their cause and prevention. Lancet 2007;370:786-96.

6 Yildiz S, Gonullu N, Yildiz BP, et al. The role of typical and atypical pathogens in acute exacerbations of chronic obstructive pulmonary disease. Clin Respir J 2021;15:209-15.

7 D'Anna SE, Maniscalco M, Cappello F, et al. Bacterial and viral infections and related inflammatory responses in chronic obstructive pulmonary disease. Ann Med 2021;53:135-50.

8 Bafadhel M, McKenna S, Terry S, et al. Acute exacerbations of chronic obstructive pulmonary disease: identification of biologic clusters and their biomarkers. Am J Respir Crit Care Med 2011;184:662-71.

9 Sethi S, Murphy TF. Infection in the pathogenesis and course of chronic obstructive pulmonary disease. $N$ Engl J Med 2008;359:2355-65.

10 Gompertz S, O'Brien C, Bayley DL, et al. Changes in bronchial inflammation during acute exacerbations of chronic bronchitis. Eur Respir J 2001;17:1112-9.

11 Davido B, Makhloufi S, Matt M, et al. Changes in eosinophil count during bacterial infection: revisiting an old marker to assess the efficacy of antimicrobial therapy. Int J Infect Dis 2017;61:62-6.

12 Calandra T, Cohen J, International Sepsis Forum Definition of Infection in the ICU Consensus Conference. The International sepsis forum consensus conference on definitions of infection in the intensive care unit. Crit Care Med 2005;33:1538-48.

13 López-Campos JL, Hartl S, Pozo-Rodriguez F, et al. Antibiotic prescription for COPD exacerbations admitted to hospital: European COPD audit. PLoS One 2015;10:e0124374.

14 Goossens H, Ferech M, Vander Stichele R, et al. Outpatient antibiotic use in Europe and association with resistance: a cross-national database study. Lancet 2005;365:579-87.

15 Cheysson F, Brun-Buisson C, Opatowski L, et al. Outpatient antibiotic use attributable to viral acute lower respiratory tract infections during the cold season in France, 2010-2017. Int J Antimicrob Agents 2021;57:106339.

16 Davies J, Davies D. Origins and evolution of antibiotic resistance. Microbiol Mol Biol Rev 2010;74:417-33. 
17 Liu B, Pop M. ARDB--Antibiotic Resistance Genes Database. Nucleic Acids Res 2009;37:D443-7.

18 Bergmans DC, Bonten MJ, Gaillard CA, et al. Indications for antibiotic use in ICU patients: a one-year prospective surveillance. $J$ Antimicrob Chemother 1997;39:527-35.

19 Sethi S. Infectious etiology of acute exacerbations of chronic bronchitis. Chest 2000;117:380s-5.

20 Hurst JR, Donaldson GC, Perera WR, et al. Use of plasma biomarkers at exacerbation of chronic obstructive pulmonary disease. Am J Respir Crit Care Med 2006;174:867-74.

21 Valent P, Klion AD, Horny H-P, et al. Contemporary consensus proposal on criteria and classification of eosinophilic disorders and related syndromes. J Allergy Clin Immunol 2012;130:607-12.

22 Rothenberg ME. Eosinophilia. N Engl J Med 1998;338:1592-600.

23 MacDonald MI, Osadnik CR, Bulfin L, et al. Low and high blood eosinophil counts as biomarkers in hospitalized acute exacerbations of COPD. Chest 2019;156:92-100.

24 Terradas R, Grau S, Blanch J, et al. Eosinophil count and neutrophillymphocyte count ratio as prognostic markers in patients with bacteremia: a retrospective cohort study. PLoS One 2012;7:e42860.

25 Abidi K, Khoudri I, Belayachi J, et al. Eosinopenia is a reliable marker of sepsis on admission to medical intensive care units. Crit Care 2008;12:R59.

26 Choi J, Oh JY, Lee YS, et al. The association between blood eosinophil percent and bacterial infection in acute exacerbation of chronic obstructive pulmonary disease. Int J Chron Obstruct Pulmon Dis 2019;14:953-9.

27 von Elm E, Altman DG, Egger M, et al. The strengthening the reporting of observational studies in epidemiology (STROBE) statement: guidelines for reporting observational studies. Lancet 2007;370:1453-7.

28 Çolak A, Yılmaz C, Toprak B, et al. Procalcitonin and CRP as biomarkers in discrimination of community-acquired pneumonia and exacerbation of COPD. J Med Biochem 2017;36:122-6.

29 Schuetz P, Christ-Crain M, Thomann R, et al. Effect of procalcitoninbased guidelines vs standard guidelines on antibiotic use in lower respiratory tract infections: the ProHOSP randomized controlled trial. JAMA 2009;302:1059-66.

30 Tanriverdi H, Örnek T, Erboy F, et al. Comparison of diagnostic values of procalcitonin, C-reactive protein and blood neutrophil/ lymphocyte ratio levels in predicting bacterial infection in hospitalized patients with acute exacerbations of COPD. Wien Klin Wochenschr 2015;127:756-63.

31 Singh D, Kolsum U, Brightling CE, et al. Eosinophilic inflammation in COPD: prevalence and clinical characteristics. Eur Respir J 2014;44:1697-700.

32 Liu A, Bui T, Van Nguyen H, et al. Serum C-reactive protein as a biomarker for early detection of bacterial infection in the older patient. Age Ageing 2010;39:559-65.

$33 \mathrm{Ni} \mathrm{W}, \mathrm{Bao} J$, Yang D, et al. Potential of serum procalcitonin in predicting bacterial exacerbation and guiding antibiotic administration in severe COPD exacerbations: a systematic review and meta-analysis. Infect Dis 2019;51:639-50.
34 Wilkinson TMA. Are inhaled corticosteroids increasing the "load" for some patients with COPD? Eur Respir J 2017;50:1701848.

35 Prentice RL, Szatrowski TP, Fujikura T, et al. Leukocyte counts and coronary heart disease in a Japanese cohort. Am J Epidemiol 1982;116:496-509.

36 Sweetnam PM, Thomas HF, Yarnell JW, et al. Total and differential leukocyte counts as predictors of ischemic heart disease: the Caerphilly and Speedwell studies. Am J Epidemiol 1997;145:416-21.

37 Shah AD, Denaxas S, Nicholas O, et al. Low eosinophil and low lymphocyte counts and the incidence of 12 cardiovascular diseases: a caliber cohort study. Open Heart 2016;3:e000477.

38 Holland M, Alkhalil M, Chandromouli S, et al. Eosinopenia as a marker of mortality and length of stay in patients admitted with exacerbations of chronic obstructive pulmonary disease. Respirology 2010;15:165-7.

39 Pavord ID, Jones PW, Burgel P-R, et al. Exacerbations of COPD. Int $J$ Chron Obstruct Pulmon Dis 2016;11 Spec Iss:21-30.

40 Kuwal A, Joshi V, Dutt N, et al. A prospective study of bacteriological etiology in hospitalized acute exacerbation of COPD patients: relationship with lung function and respiratory failure. Turk Thorac $J$ 2018;19:19-27.

41 Sethi S. Bacteria in exacerbations of chronic obstructive pulmonary disease: phenomenon or epiphenomenon? Proc Am Thorac Soc 2004;1:109-14.

42 Gaude GS, Rajesh BP, Chaudhury A, et al. Outcomes associated with acute exacerbations of chronic obstructive pulmonary disorder requiring hospitalization. Lung India 2015;32:465-72.

43 Vollenweider DJ, Frei A, Steurer-Stey CA, et al. Antibiotics for exacerbations of chronic obstructive pulmonary disease. Cochrane Database Syst Rev 2018;10:Cd010257.

44 Viniol C, Vogelmeier CF. Exacerbations of COPD. Eur Respir Rev 2018;27. doi:10.1183/16000617.0103-2017. [Epub ahead of print: 31 Mar 2018].

45 Brusse-Keizer MGJ, Grotenhuis AJ, Kerstjens HAM, et al. Relation of sputum colour to bacterial load in acute exacerbations of COPD. Respir Med 2009;103:601-6.

46 Llor C, Bjerrum L, Munck A, et al. Predictors for antibiotic prescribing in patients with exacerbations of COPD in general practice. Ther Adv Respir Dis 2013;7:131-7.

47 Koutsokera A, Stolz D, Loukides S, et al. Systemic biomarkers in exacerbations of COPD: the evolving clinical challenge. Chest 2012;141:396-405

48 Nyström PO. The systemic inflammatory response syndrome: definitions and aetiology. J Antimicrob Chemother 1998;41 Suppl A:1-7.

49 McNally M, Curtain J, O'Brien KK, et al. Validity of British thoracic Society guidance (the CRB-65 rule) for predicting the severity of pneumonia in general practice: systematic review and meta-analysis. Br J Gen Pract 2010;60:e423-33.

50 Edwards L, Perrin K, Wijesinghe M, et al. The value of the CRB65 score to predict mortality in exacerbations of COPD requiring hospital admission. Respirology 2011;16:625-9. 